\title{
Rooftop Garden and Lighting Automation by the Internet of Things (IoT)
}

\author{
Shahajan Miah, S. M. Rakib Raihan, Md. Mahmudul Hasan Sagor, Md. Mahmud Hasan, \\ Debasish Talukdar, Shahnewas Sajib, Md. Rakibul Alam Naim, Ummay Anisa, and Ummay Suaiba
}

\begin{abstract}
The Internet of Things (IoT) enables individuals to easily operate more than only digital things through the internet using a user-friendly GUI. The researchers are particularly interested in the use of IoT for household and industrial automation, as well as the monitoring of a variety of physical properties over the internet. This internet of things project showcased current system upgrades as well as creative solutions to specific issues. The Internet of Things (IoT) is a technology that allows for continuous monitoring of wirelessly linked objects by utilizing wireless sensor networks, nanostructures, and scalability.The Internet of Things is made up of many physical objects that connect with one another. It would allow users to approach numerous applications in unique ways, such as smart homes and smart agriculture, by enabling seamless connection between virtual and actual equipment, assuring dialogues at any time and from any location. The user may examine the status of any network resources. The IoT being used has its own network plan, allowing it to observe the state of embedded devices and take appropriate action by passing instructions regulating the objects via the web server utilizing Internet connections. This project aimed to create a rooftop home gardening facility capable of synchronizing the utility grid with a moisture sensor and a water pump. We were able to effectively build the central notion of the synchronization technique and connect it to a mobile device through Bluetooth. Several tweaks and trial and error tactics were utilized to make this one function. We have just shown the procedure of synchronizing and switching approaches in this study.
\end{abstract}

Key words - IoT, Arduino Nano, Moisture Sensor, LDR, Relay, Microcontroller IC ATmega328P, Water Pump.

\section{INTRODUCTION}

Home automation is a method of managing and monitoring home appliances and features using a computer or smartphone. Sustaining and retaining linked to everyday home systems with a phone or tablet can significantly increase quality of life. It is more than simply remotely measuring and managing the lights, fan, gas leaks, motion detection, and watering the garden from any location using a cell phone. Pleasure, flexibility, cost reductions, and protection may all be afforded by a smart house. Many people lose interest in gardening since it requires a lot of labor to maintain the seedlings healthy.As a result, an automated and quantified plant care system might be a useful tool to

Submitted on December 16, 2021.

Published on January 20, 2022

Shahajan Miah, Department of EEE, Bangladesh University of Business $\&$ Technology, Bangladesh.

(e-mail: miahbubt@ bubt.edu.bd).

S. M. Rakib Raihan, Department of EEE, Bangladesh University of Business \& Technology, Bangladesh.

Md. Mahmudul Hasan Sagor, Department of EEE, Bangladesh University of Business \& Technology, Bangladesh.

Md. Mahmud Hasan, Department of EEE, Bangladesh University of Business \& Technology, Bangladesh. maintaining the gardening in the rooftop of the building. The Internet of Things (IoT) is a new technology that leverages the internet to include electronic/mechanical bias, autos, and other tangible biases that are connected to the internet. We are currently developing a system that consists of a dispersed wireless network of soil moisture, temperature, and ultrasonic sensors. To transport water from a tank controlled by a gate, the project required the use of sensors, an Arduino, and pipes. A water level sensor was installed near the roots, while a daylight sensor was installed further away to detect sunbeams.

These detectors provide data to the Arduino for analysis. There will also be a Bluetooth gadget that links the Android device to an app that allows the user to control the automated task manually. The primary goal of this design is to preserve water while increasing the output of trees and stores in the theater by covering the expansion of businesses. We created a system that uses Arduino-controlled sensor to autonomously sprinkle an exact amount of water based on soil humidity, sunshine intensity, and water location. For smooth growth, each type of industry requires a distinct level of soil humidity. As a result, soil moisture is a significant element that can influence the amount of water required. Furthermore, a tree requires a certain quantity of sunlight. So, watering at the incorrect moment may cause more harm than benefit.

\section{OBJECTIVE}

The major goal of this project was to create a gadget that would allow us to regulate the water level and lighting of a rooftop garden using IOT.

\section{BACKGROUND}

The notion of a smart house roof garden watering system is gaining popularity by incorporating a variety of functions. The system in [1] is powered by Cortex-A 8 and ZigBee. The system is split into three parts: a centralized intelligent control interface, a connected gateway node, and a Zigbee communication network in the home. It can do things like safety and alarm, inner topography evaluation, steps must be

Debasish Talukdar, Department of EEE, Bangladesh University of Business \& Technology, Bangladesh.

Shahnewas Sajib, Department of EEE, Bangladesh University of Business \& Technology, Bangladesh.

Md. Rakibul Alam Naim, Department of EEE, Bangladesh University of Business \& Technology, Bangladesh.

Ummay Anisa, Department of EEE, Bangladesh University of Business \& Technology, Bangladesh.

Ummay Suaiba, Department of EEE, Bangladesh University of Business \& Technology, Bangladesh. 
completed electrical item control, and sophisticated lighting. Drug addicts can pierce the Internet web to cover the most expensive house furnishings ever. Drug abusers can also engage with a home furnishing gadget at any time by using a mobile phone with Android smart furnishing control client operations.

In [2,] the system includes Intel Galileo to combine wired networking and wireless connectivity in order to offer the consumer with remote control over their house's multicolored lights, suckers, and appliances while also saving data in the dark. The system will change on its own based on sensor data. This system is designed to be low-cost and expandable, allowing it to operate a large variety of devices. The innovative system monitors detector data such as temperature, gas, light, and stir detectors and activates a process based on the requirement. When it gets dark in the morning, for example, it is turned off. It also instantaneously saves the detector parameters in the pall (Gmail). The individual will be able to evaluate the condition of colored variables in the house at any time and from any location.

In [3,] depicts operating systems such as iOS building automation system that allows many users to control the appliances via an Android platform or a website. The system consists of three equipment: a local unit that transmits signals to household appliances, a web server that keeps customer records and assists the other components, and a mobile smart device that runs an Android app. Google's distributed cloud systems and services are used to facilitate messaging between the parts. The Google's distributed cloud platforms and services are used to facilitate messages between the components. The solution is cost-effective since the service and data dissemination are designed to be public and free on Google platforms. To monitor irrigation operations, the model in [4] incorporates soil moisture, temperature, and pressure sensors. When a system failure occurs, such as when pipes break or emitters become blocked, we investigate it thoroughly. Furthermore, in order to achieve high QoS performance, we identify two primary traffic levels for the information carried by the WSAN and use an appropriate priority-based routing protocol. Simulations on the NS-2 simulator yield encouraging latency and Packet Delivery Ratio (PDR) results, particularly for priority traffic.

In [5] describes a system that involves a large wife network of moisture in the soil and temperature sensors distributed in plant root layers. The gateway device analyzes sensor data, engages actuators, and communicates information to a web application. An algorithm has been developed to manage water quantity using sensor threshold values input into a microcontroller-based gateway. The study field of sensor networks strength time is a major problem that may be handled by applying ZigBee technology in [6]. The primary purpose is to comprehend how data travels across wireless media transmission using WSN and a monitoring system. Because temperature, soil moisture, and air humidity are the most critical variables to manage in $\mathrm{PA}$, the design of an irrigation canal is mechanized by utilizing adjustable characteristics like as these (Precision Agriculture). To predict real-time local weather features, ref. [7] proposes a real-time spatial interpolation technique based on surrounding ASW. It also includes a corrective approach that makes use of sparse WSN with an integrated soil moisture sensor.

\section{THEORY OF THE PROJECT}

\section{A. Arduino Nano}

Arduino is associate degree ASCII text file natural philosophy prototyping platform supported flexible, easy hardware and software system. it's aimed towards artists, designers, potters, and anybody else curious about making interactive things or environments. By inputting data from colorful detectors, Arduino may smell the terrain and influence its environment by activating lights, motors, and other selections. Arduino systems will operate alone or in conjunction with computer code running on a pc (e.g., Flash, Processing, MaxMSP). The Arduino Nano could be a face mount bread board embedded interpreter with associate integrated USB port. it is the most cost-effective, most comprehensive, and most breadboard-friendly. It includes all of the options of the Diecimila/Duemilanove (electrically), and further associate Logue input legs and an intrinsically $5 \mathrm{~V}$ AREF dress. It lacks an influence jack physically. The Nano is mechanically detected associated switched to an improved implicit power supply.

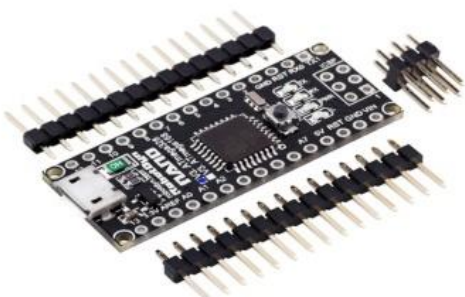

Fig. 1. Arduino Nano.

\section{B. Microcontroller IC ATmega 328P}

The top quality Chips pico Power 8- bit AVR RISCrestricted machine integrates $32 \mathrm{~KB}$ ISP compact flash with read- at the same time as capabilities, 1024B EEPROM, 2KB SRAM, twenty three general purpose I/ O lines, thirty two general purpose operating registers, 3 versatile timekeeper/ counters with compare modes, internal and external interrupts, periodical programmable USART, a byteacquainted 2- line diurnal interface, SPI periodic harborage, a The device's voltage vary is one.8-5.5 volts. By process essential directions in an exceedingly single timer cycle, the device delivers outputs that correspond to one unit of measurement per megacycle per second whereas equalization power consumption and refinement speed.

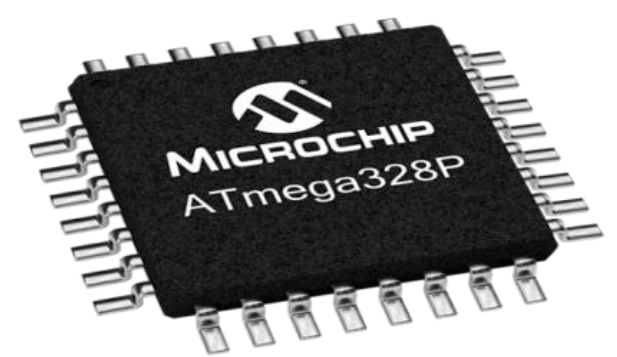

Fig.2. Microcontroller IC ATmega 328P 


\section{Water Pump}

Submersible pump motors that can operate on two AA batteries are an option for this tank. Power supply of 5-6 volts. This pump has a maximum flow rate of 120 liters per hour and an average current draw of $220 \mathrm{~mA}$ at all times. It's as simple as submerging the tube pipe into the motor outlet and turning it on. The water should always be ahead of the engine. Practicing might cause engine damage due to overheating. a tiny DC 3-6V Submersible Pump will also be built. Mini pump for Fountain Garden's miniature water circulation system is a do-it-yourself project.
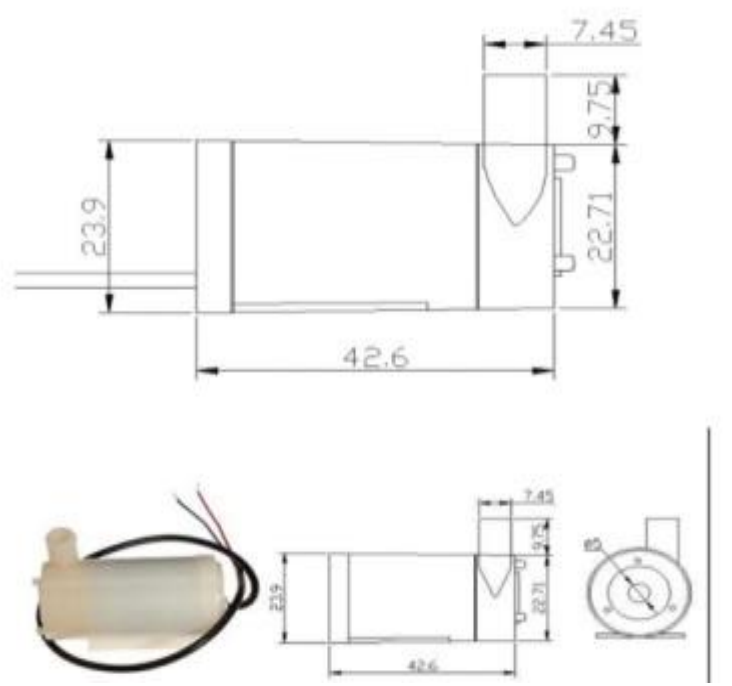

Fig. 3. Schematic diagram of submersible pump mini.

\section{Moisture Sensor}

In order to protect the metal from corrosion, Immerse Gold is employed in this Sensing Element. PCBs with large BGA packages benefit greatly from ENIG because of its excellent polarization, corrosion resistance, and ability to function with unsealed contact shells like membrane switching devices and points of contact. ENIG is more expensive than conventional (and less expensive) face platings like HASL (solder). Using this Humidity Detector, you can measure the quantity of moisture in the soil. Low-tech sensors like this one are great for monitoring water levels in urban gardens or for keeping an eye on your pet plants.

Using this Wetness Sensor, you can tell whether or not there is water nearby, which gives your lawn's flora a way to ask for help from you. Simply put it into the soil and do an analysis. In theory, with this sensor, you should be able to get the plant to tell you that it's thirsty and ask for a few drinks. In order to assess the humidity level, this sensor uses two probes to skip through soil at a constant speed and then measures the resistance. The soil's energy behavior improves fast (with much reduced resistance) when there is more water in the soil, but dry soil transfers energy poorly (more excellent resistance). You could find it handy to remind you to water your indoor plants or to show the soil moisture on your yard. For this sensor, the 10 Expansion Shield provides the best protection. One of the most essential aspects of this module is its low power consumption and great sensitivity. Arduino UNO Arduinomega 2560's ADK, and so on, might be compared to this product's features.

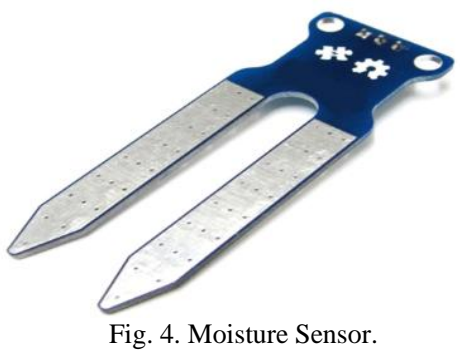

\section{E. Bluetooth Module HC-05}

Wireless serial connections may be established using the HC-05 Bluetooth SPP (Serial Port Protocol) module. 2.4GHz radio transceiver and baseband are included in the Bluetooth V2.0+EDR (Enhanced Knowledge Rate) 3Mbps Modulation Port Bluetooth module. An external single-chip CMOS/AFH Bluetooth module from CSR is used (Adaptive Frequency Hopping Feature). In terms of size, it measures $12.7 \times 27 \mathrm{~mm}$. I'm hoping it'll have an impact on the whole process of designing and developing new products.

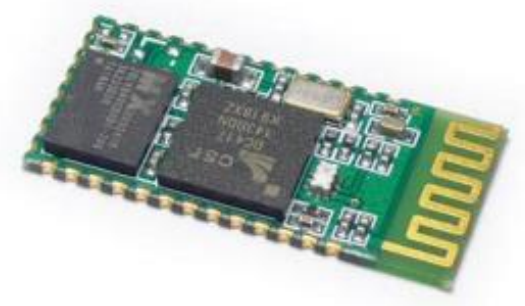

Fig. 5. Bluetooth HC-05.

\section{F. Relay}

The most common electrical component is the relay. Consider how we may use one in our circuits to suit our project's requirements. First, we need to look at two important relay variables before we begin the driving course. In order for the relay to be activated, it must be able to flip the contact from Common> NC to Common> NO at the proper voltage. Depending on the voltage you have available in your design, you may pick a relay with a trigger voltage as low as $3 \mathrm{~V}$, as high as $6 \mathrm{~V}$, or as low as $12 \mathrm{~V}$. For a dc power source, the maximum voltage and current that the relay's $\mathrm{NC}$, NO, or joint terminal can handle is $30 \mathrm{~V}$ and $10 \mathrm{~A}$; this value is also a variable. Make sure the load you're using falls within this range.

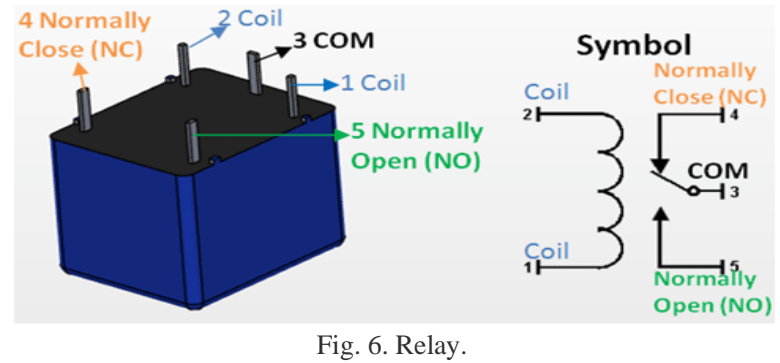

\section{G. $L D R$}

As soon as light levels fall below a certain threshold, the resistance drops to a few thousandth of a milliohm, depending 
on the model. LED resistors are available in a broad variety of shapes and hues. Alarms, switch devices, clocks, streetlights, and other electrical circuits all employ LDRs, which are small, low-power, and versatile. Audio limiters and compressors, for example, make use of the square measure. It is common practice to activate or deactivate a tool in line with the heaviest object in the immediate vicinity.

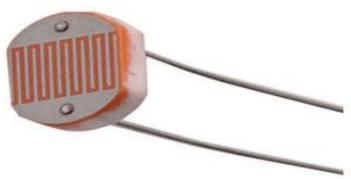

Fig. 7. LDR.

\section{DESIGN AND FABRICATION}

\section{A. Block Diagram}

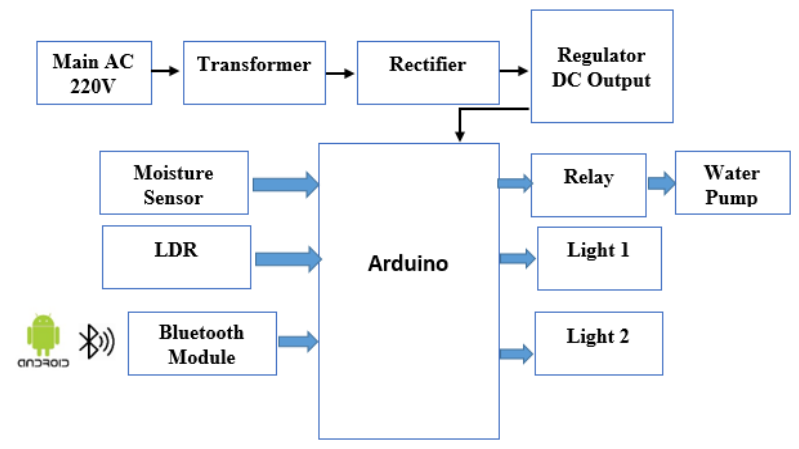

Fig. 8. Block Diagram,

\section{B. Circuit Description}

Atmega 328P microprocessor is the heart of the ArduinoNano (Atmega 328P). Regulated 5V power is supplied via a 7805 regulator IC, which is controlled by an external resistor and capacitor. Another important part of this device is the Bluetooth module (HC05), which enables wireless communication between microcontrollers IC and mobile phones.

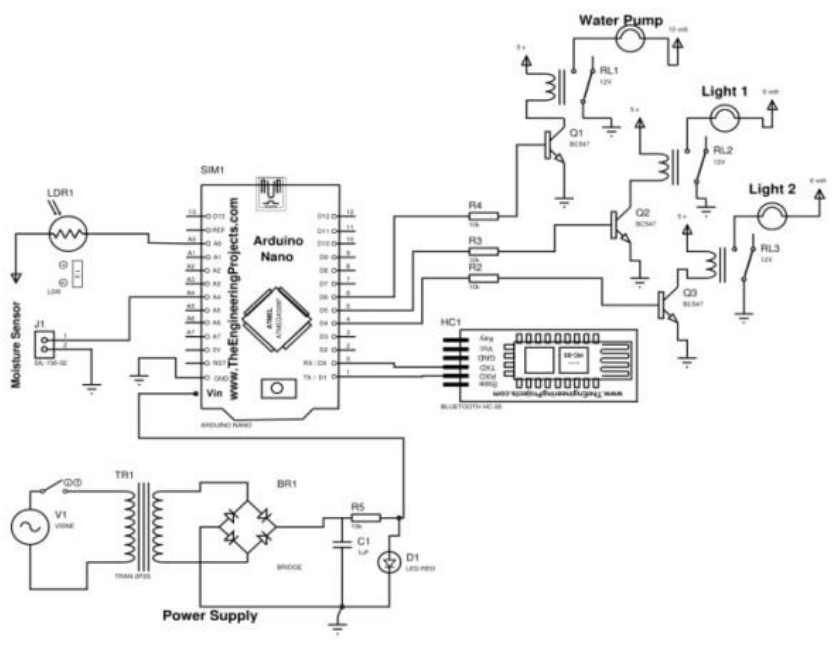

Fig. 9. Circuit Diagram.

\section{Working Principle}

Soil moisture is detected with the Moist Sensor. When we're below our expected ratio, the water pump will generate water for the rooftop garden. The LDR sensor can also tell whether it's day or night using the information it gathers. During the night, the LDR is open, and the lights are on.

\section{Arduino Software}

It is possible to program the Arduino Uno microcontroller using the Arduino software. There is no need to install any additional software other than Arduino. At this point you'll want to access the Tools, Board menu and choose "Arduino Uno" (according to the microcontroller on your board). You don't need to use an external hardware programmer to update the Arduino Uno's ATmega328 chip, which is used in this board. The chip is preprogrammed with a boot loader that makes this possible.

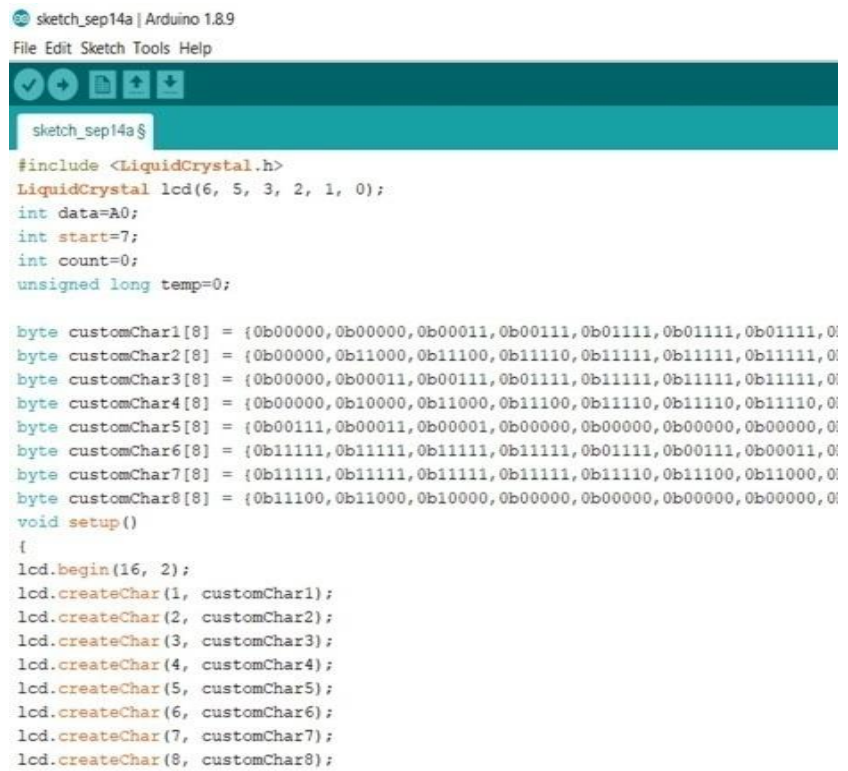

Fig. 10. Arduino Software Interface IDE.

The communication protocol of choice is the original STK500 (reference, C header files). Programming the microcontroller through the header's ICSP port is also an option (In-Circuit Serial Programming). The microcode ASCII text file for the ATmega16U2 microcontroller is provided (or $8 \mathrm{U} 2$ within the rev1 and rev2 boards). There is a DFU boot loader on the ATmega16U2/8U2. On Rev1 panels, this is done by connecting the solder jumper (located next to a map of Italy) and restarting 8U2. Rev2 and subsequent boards: In order to enter DFU mode, a resistance pulls the HWB line 8U2/16U2 down. One of the best clever microprocessors on the market today, the Arduino Uno has extra choices for interfacing with a laptop, another Arduino, or replacement microcontrollers. Digital pins 0 - (RX) for receiving data and pin no. 1 (TX) for transmitting data are accessible on the ATmega328's UART TTL at $5 \mathrm{~V}$ serial connection. In order to communicate with the computer, a USB-connected ATmega16U2 on the panel provides serial connection. A high-quality USB COM driver is used in the 16U2 code so that there is no need for additional drivers.

It's necessary to have an.inf file on Windows in order to run the program properly. Data may be sent and received 
from and to the Arduino board using the serial monitor included in this package. LEDs on the RX and Texas boards on the USB-to-serial chip and USB connection may flash after data has been sent (but not for serial communication on pins zero and 1). It's a program SPI communication is possible on any of the Uno's digital pins that may be connected to a computer using this serial library. Additional connectivity options include I2C (TWI) and SPI. The I2C bus is made simpler to use by the Arduino software's Wire library. Programs developed in the Arduino programming language, $\mathrm{C}$ or $\mathrm{C}++$, are known as sketches. avrdude is used to upload the software to the AVR using the GNU toolchain and AVR Libc.

\section{E. Proteus Software}

For electronic style automation, the Proteus design Suite is a proprietary computer code tool. Creating schematics and electronic prints for the fabrication of computer circuit boards is a common task for electronic design engineers and technicians who work with computer code. In 1988, John Jameson, head of the firm, created the first version of the Proteus style Suite for DOS, known as PCB. In 1990, support for Schematic Capture was implemented, and a move to Windows happened soon after.

In 1996, Proteus added support for blended SPICE simulation, and in 1998, it added support for microcontroller simulation. 3D Board visualization, based on shape-based auto-routing, was another significant product upgrade in 2006. MCAD import/export and an advanced simulation IDE were also implemented in 2011 and 2015, respectively. Support for fast-paced fashion was another 2017 trend. In contrast to feature-driven product releases, maintenancebased service packs are given on a more ad-hoc schedule.

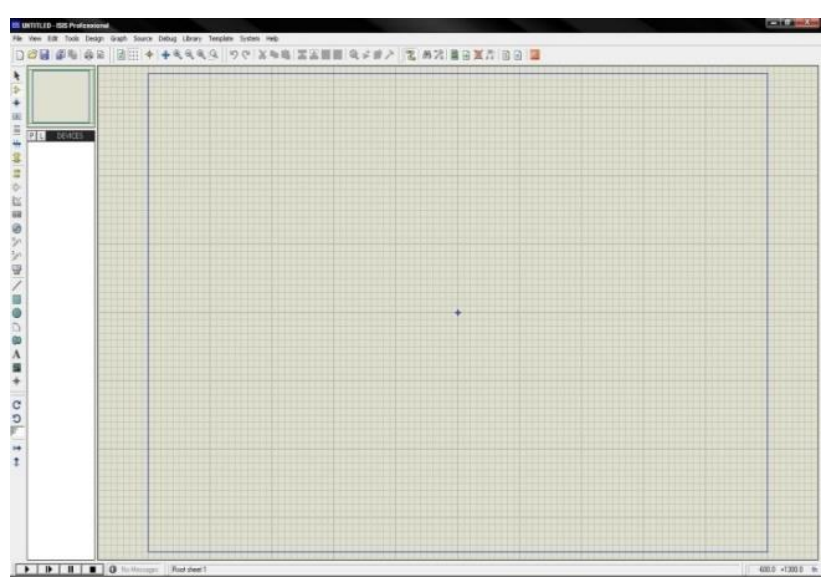

Fig. 11. Proteus Software Interface.

\section{F. Microcontroller Simulation}

A hex file or a corrected hex file is applied to the microcontroller portion of the schematic in Proteus's microcontroller simulation. As a result, it has been combined with any analog and digital physical scientific research. A variety of project prototype applications are therefore possible, including control, thermal management, and computer program development. Because it doesn't need any special gear, it's a popular choice among amateurs. It may also be utilized as a coaching or teaching tool. Co-simulation is supported for the following:
- Microcontrollers from Microchip Devices, including the PIC10, PIC12, PIC16, PIC18, PIC24, and dsPIC33;

- Embedded systems Atmel AVR (and Arduino), 8051, and ARM Cortex-M3;

- Microcontroller based NXP 8051, ARM7, ARM Cortex$\mathrm{M} 0$, and ARM Cortex-M3;

- Microcontrollers from Texas Instruments MSP430, PICCOLO DSP, and ARM Cortex-M3;

- Parallax Basic Stamp, HC11 Freescale, and 8086 Microcontrollers.

\section{G. PCB Design}

The PCB module receives a net list automatically generated by the schematic capture module. In addition to the user-specified design principles and design automation tools, it utilises this information to help in the construction of errorfree panels. Designing PCBs with more than 16 copper formations is restricted by a product configuration.

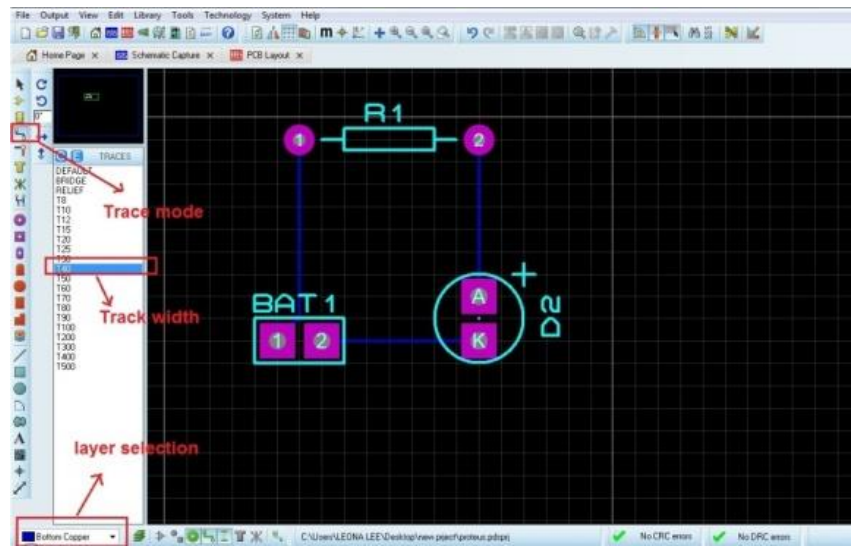

Fig. 12. PCB Design.

\section{H. 3D Verification}

Allows a semi-transparent height plane to be used to simulate the container for 3D viewing of the board under development. An CAD program like SolidWorks or Autodesk will be used to put the board in the correct position after obtaining the STEP result.

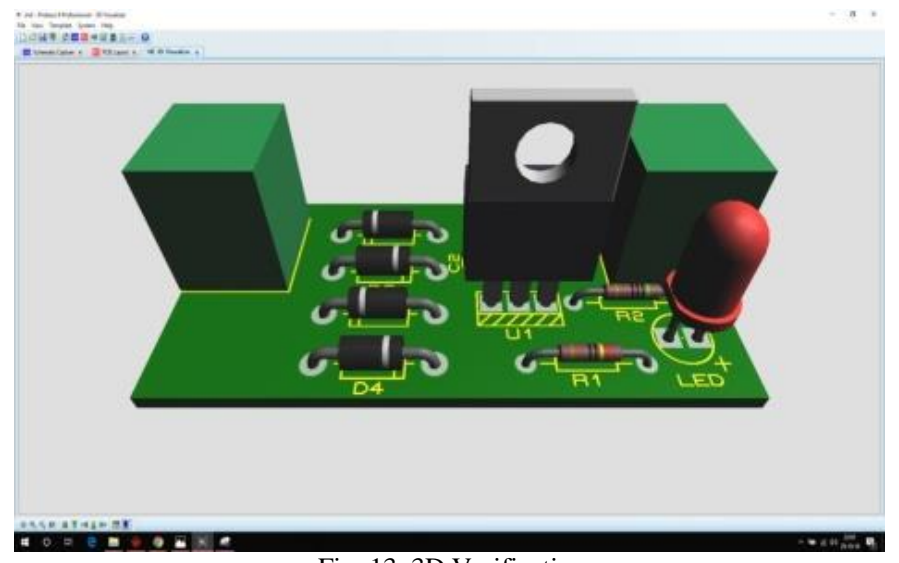

Fig. 13. 3D Verification.

\section{RESULTS}

The proposed technique comprises of a large number of sensors, including moisture and light detection and reflection 
(LDR). Initial connections are made between the Arduino and sensor, with the possibility of connecting through Bluetooth on mobile devices. This enables anybody to manually start the water pump and switch on or off the lights. Our project will begin by detecting the sun using an LDR sensor for the purpose of monitoring the farm; when night falls, the LDR sensor will turn on the lights in the building. If the number is less than or equal to 700, the Moisture Sensor will detect the water level in the soil, indicating that the water level is in a desirable position; however, if the value is more than or equal to 900 , the Moisture Sensor will begin automatically delivering water. The water pump will be linked to the Arduino and programmed with a delay time for dry soil to ensure that it operates properly.

VII. COST ESTIMATION

TABLE I: CoST ESTIMATION OF THE DEVICE

\begin{tabular}{|c|c|c|c|c|c|}
\hline $\begin{array}{l}\text { S.1. } \\
\text { No }\end{array}$ & Particulars & Specification & Qty. & $\begin{array}{c}\text { Unit } \\
\text { Price } \\
\text { (Taka) }\end{array}$ & $\begin{array}{c}\text { Total } \\
\text { Price } \\
\text { (Taka) }\end{array}$ \\
\hline 1 & Arduino Nano & Atmega 328p & 1 & 280 & 280 \\
\hline 2 & LDR & & 1 & 200 & 200 \\
\hline 3 & $\begin{array}{l}\text { Moisture } \\
\text { Sensor }\end{array}$ & & 1 & 120 & 120 \\
\hline 4 & Water Pump & $\begin{array}{c}3- \\
9 \mathrm{v}, 400 \mathrm{mAh}\end{array}$ & 1 & 300 & 300 \\
\hline 5 & DC Gear Motor & $5 \mathrm{v}, 400 \mathrm{mAh}$ & 1 & 120 & 120 \\
\hline 6 & $\begin{array}{l}\text { Voltage } \\
\text { Regulator }\end{array}$ & LM 7805 & 1 & 7 & 7 \\
\hline 7 & Transformer & $\begin{array}{c}\text { 220/12V, } \\
\text { 3amps }\end{array}$ & 2 nos & 350 & 700 \\
\hline 8 & Bridge & 2W10 & 1 & 6 & 6 \\
\hline 9 & Capacitor & $1000 \mu \mathrm{f}, 25 \mathrm{~V}$ & 1 & 8 & 8 \\
\hline 10 & Relay & 4 channel & 1 & 250 & 250 \\
\hline 11 & $\begin{array}{c}\text { Bluetooth } \\
\text { Module HC-05 }\end{array}$ & & 1 & 250 & 250 \\
\hline \multicolumn{5}{|c|}{ Total (BDT) } & 2241 \\
\hline \multicolumn{5}{|c|}{ Total (USD) } & 26.36 \\
\hline
\end{tabular}

\section{CONCLUSION}

The goal of this investigation is to determine how the moisture sensor determines the presence or absence of water. We also include a daylight sensor, which is powered by an Arduino Nano and is used to control the garden's lighting system at night. When the water level falls below a specific threshold, we design our Water Pump to manually communicate with the pump through Bluetooth and Arduino, causing the pump to start producing water. This project develops an effective technique of electrifying or producing electricity via the use of an energy-collecting circuit. Finally, we were able to complete our project successfully.

\section{ACKNOWLEDGMENT}

All thanks to "Almighty Allah," the most compassionate, the most gracious, the wellspring of knowledge and wisdom bestowed upon humankind, who bestowed upon us the strength, mind, and aptitude to embark on this incredible voyage of discovery. We are grateful to Prof. Dr. Md. Fayazz Khan, Vice-Chancellor, Bangladesh University of Business and Technology (BUBT) and Prof. Dr. Md. Anwar Hossain, Chairman, Department of EEE, Bangladesh University of
Business and Technology, for allowing us to use the facilities of the EEE Department to make our system and for all his advice and suggestions.

\section{CONFLICT OF INTEREST}

We declare that there is no conflict of interest in our research work.

\section{REFERENCES}

[1] GargiRajadhyaksha, SiddharthMody, SnehaVenkateswar, Portable Text to Speech Convertor, International Journal of Emerging Technology and Advanced Engineering (IJETAE), August 2013;3(8).

[2] Smt.M.Baby, P.Harini, M.Sailaja, K.AnnieSumantha. SMS based Wireless E-Notice Board, International Journal of Emerging Technology and Advanced Engineering (IJETAE), March 2013;3(3).

[3] Jigyasa Mishra, ApoorvSrivastav, Rahul Jain, Arduino Based LCD Display, International Journal of Emerging Technology and Advanced Engineering (IJETAE), June 2014; 3(5).

[4] https://learn.sparkfun.com/tutorials/bluetooth-basics.

[5] https://en.wikipedia.org/wiki/Bluetooth.

[6] http://electronics.howstuffworks.com/bluetooth1.htm

[7] https://www.bluetooth.com/what-is-bluetoothtechnology/bluetoothtechnology-basics.

[8] http://www.creativeworld9.com/2011/04/abstract-andfull-paper-onbluetooth.html.

[9] http://www.slideshare.net/kashyapshah11/bluetooth.

[10] http://m.instructables.com/id/Arduino-AND-BluetoothHC-05Connecting-easily/?ALLSTEPS

[11] http://www.techbitar.com/modify-the-hc-05-bluetoothmoduledefaults-using-at commands.html.

[12] E. Ferro and F. Potorti, Bluetooth and Wi-Fi wireless protocols: a survey and a comparison, Wireless Communications, IEEE, February 2005;12(1):12-26.

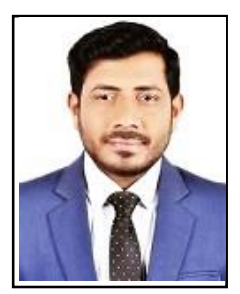

Shahajan Miah was born on November 11, 1987 in Jagannthpur, Sunamganj, Sylhet, Bangladesh. He is an Assistant Professor, Dept. of EEE, Bangladesh University of Business \& Technology (BUBT), Dhaka, Bangladesh. He completed his Master of Science and B.Sc (Hon's) from Shahjalal University of Science \& Technology (SUST), Sylhet, Bangladesh. He published a book (Basics in Natural Sciences) and several research articles in the different international journals. It is worth mentioning that he is a member of the Bangladesh Physical Society.

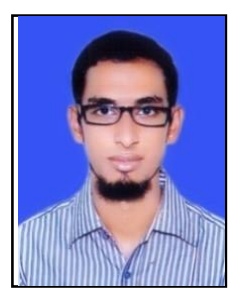

S.M. Rakib Raihan, Lecturer, Dept. of EEE Bangladesh University of Business \& Technology (BUBT), Dhaka, Bangladesh. He completed his B.Sc. in EEE from International University of Technology (IUT), Dhaka, Bangladesh.

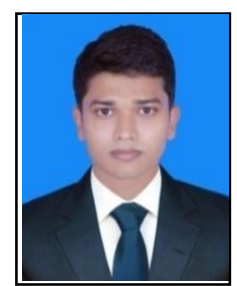

Md. Mahmudul Hasan Sagor, Lecturer, Dept. of EEE, Bangladesh University of Business \& Technology (BUBT), Dhaka, Bangladesh. He completed his B.Sc. in EEE from Khulna University of Engineering \& Technology (KUET), Khulna, Bangladesh. 


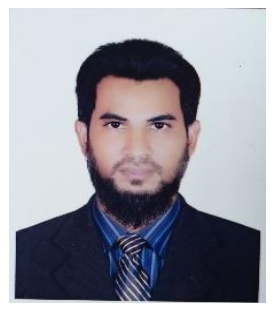

Md. Mahmud Hasan, Lecturer, Dept. of EEE, Bangladesh University of Business \& Technology (BUBT), Dhaka, Bangladesh. He completed his Master of Philosophy from the Bangladesh University of Engineering \& Technology \& Master of Science and B.Sc (Hon's) from Shahjalal University of Science \& Technology (SUST), Sylhet, Bangladesh.

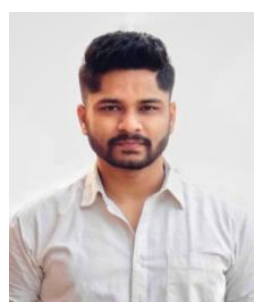

Debasish Talukdar completed his B.Sc. in EEE from Bangladesh University of Business \& Technology, Dhaka, Bangladesh.

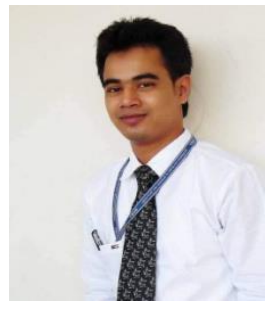

Shahnewas Sajib completed his B.Sc. in EEE from Bangladesh University of Business \& Technology, Dhaka, Bangladesh.

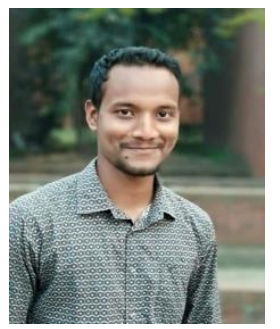

Md. Rakibul Alam Naim completed his B.Sc. in EEE from Bangladesh University of Business \& Technology, Dhaka, Bangladesh.

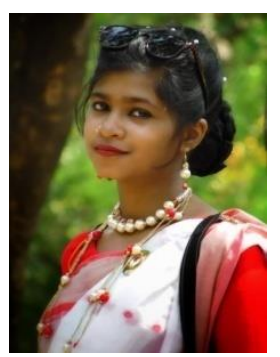

Ummay Anisa completed her B.Sc. in EEE from Bangladesh University of Business \& Technology, Dhaka, Bangladesh.

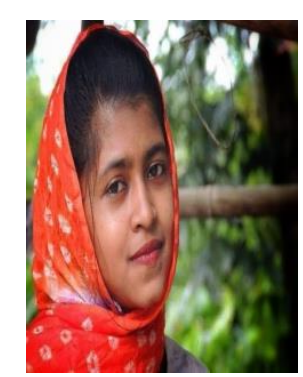

Ummay Suaiba completed her B.Sc. in EEE from Bangladesh University of Business \& Technology, Dhaka, Bangladesh. 